\title{
Inequalities for doubly nonnegative functions
}

\author{
Alexander Sidorenko \\ Rényi Institute \\ Budapest, Hungary \\ sidorenko.ny@gmail.com
}

Submitted: Aug 16, 2019; Accepted: Jan 31, 2021; Published: Feb 12, 2021

(C) The author. Released under the CC BY-ND license (International 4.0).

\begin{abstract}
Let $g$ be a bounded symmetric measurable nonnegative function on $[0,1]^{2}$, and $\|g\|=\int_{[0,1]^{2}} g(x, y) d x d y$. For a graph $G$ with vertices $\left\{v_{1}, v_{2}, \ldots, v_{n}\right\}$ and edge set $E(G)$, we define

$$
t(G, g)=\int_{[0,1]^{n}} \prod_{\left\{v_{i}, v_{j}\right\} \in E(G)} g\left(x_{i}, x_{j}\right) d x_{1} d x_{2} \cdots d x_{n} .
$$

We conjecture that $t(G, g) \geqslant\|g\|^{|E(G)|}$ holds for any graph $G$ and any function $g$ with nonnegative spectrum. We prove this conjecture for various graphs $G$, including complete graphs, unicyclic and bicyclic graphs, as well as graphs with 5 vertices or less.
\end{abstract}

Mathematics Subject Classifications: 05C35, 05C22, 26D20

\section{Introduction}

Let $\mu$ be the Lebesgue measure on $[0,1]$. Let $\mathcal{H}$ denote the space of bounded measurable real functions on $[0,1]^{2}$, and $\mathcal{G} \subset \mathcal{H}$ denote the subspace of symmetric functions. Let $\mathcal{H}_{+}$ and $\mathcal{G}_{+}$denote the subsets of nonnegative functions in $\mathcal{H}$ and $\mathcal{G}$, respectively.

Let $G$ be a simple graph with vertices $\left\{v_{1}, v_{2}, \ldots, v_{n}\right\}$ and edge set $E(G)$. We would like to know what conditions on $G$ and $g \in \mathcal{G}_{+}$guarantee that

$$
t(G, g) \stackrel{\text { def }}{=} \int_{[0,1]^{n}} \prod_{\left\{v_{i}, v_{j}\right\} \in E(G)} g\left(x_{i}, x_{j}\right) d \mu^{n} \geqslant\left(\int_{[0,1]^{2}} g d \mu^{2}\right)^{|E(G)|} .
$$

One approach is to ask what graphs $G$ satisfy (1.1) for every function $g \in \mathcal{G}_{+}$. It is easy to show that such graphs can not have odd cycles, so only graphs with chromatic number 2 are suitable candidates. It led to 
Conjecture 1.1 ([21, 22]). Let $H$ be a bipartite graph with two vertex sets $V=\left\{v_{1}, v_{2}\right.$, $\left.\ldots, v_{n}\right\}, W=\left\{w_{1}, w_{2}, \ldots, w_{m}\right\}$ and edge set $E(H) \subseteq V \times W$. Then for any function $h \in \mathcal{H}_{+}$(not necessarily symmetric)

$$
t(H, h) \stackrel{\text { def }}{=} \int_{[0,1]^{n+m}} \prod_{\left(v_{i}, w_{j}\right) \in E(H)} h\left(x_{i}, y_{j}\right) d \mu^{n+m} \geqslant\left(\int_{[0,1]^{2}} h d \mu^{2}\right)^{|E(H)|} .
$$

We discuss Conjecture 1.1 in Section 3.

For a (simple or bipartite) graph $G$, let $E(G)$ denote its edge set, and e $(G)=|E(G)|$. For a simple graph $G$, let $V(G)$ denote its vertex set, and $\mathrm{v}(G)=|V(G)|$.

The 1-subdivision of a simple graph $G$ is a bipartite graph $H=\operatorname{Sub}(G)$ with vertex sets $V(G)$ and $E(G)$, where $v \in V(G)$ and $e \in E(G)$ form an edge in $H$ if $v \in e$ in $G$.

We call a bipartite graph $H$ symmetric if it has an automorphism $\phi$ which switches its vertex-sets $V$ and $W: \phi(V)=W, \phi(W)=V$.

We call a function $g \in \mathcal{G}_{+}$doubly nonnegative if there is a function $h \in \mathcal{H}$ such that $g(x, y)=\int_{[0,1]} h(x, z) h(y, z) d \mu(z)$. Equivalently, a doubly nonnegative function is a nonnegative symmetric function with nonnegative spectrum. We call a function $g \in \mathcal{G}_{+}$completely positive if there is a function $h \in \mathcal{H}_{+}$such that $g(x, y)=\int_{[0,1]} h(x, z) h(y, z) d \mu(z)$. The terms "doubly nonnegative" and "completely positive" come from matrix theory; there exist functions which are doubly nonnegative but not completely positive (see Section 2).

In this article, we study two problems: (a) what functions $g \in \mathcal{G}_{+}$satisfy $t(G, g) \geqslant$ $\|g\|^{\mathrm{e}(G)}$ for all simple graphs $G$ (we call such functions nice); and (b) what graphs $G$ satisfy the same inequality for any doubly nonnegative function $g$ (we call such graphs good).

If $G$ is good, then Conjecture 1.1 holds for $H=\operatorname{Sub}(G)$. We show in Section 3 that for a fixed $G$, inequality (1.1) holds for any completely positive function $g$ if and only if Conjecture 1.1 holds for $H=\operatorname{Sub}(G)$. Thus, it is reasonable to expect that all completely positive functions are nice.

Conjecture 1.2. All doubly nonnegative functions are nice. All simple graphs are good.

Our Theorem 2.1 demonstrates that there are nice functions which are not doubly nonnegative.

If chromatic number $\chi(G)=2$, then goodness of $G$ should follow from Conjecture 1.1. In Sections 4-7, we give examples of good graphs $G$ with $\chi(G) \geqslant 3$. In particular, we prove that complete graphs, graphs whose complements consist of disjoint edges, unicyclic and bicyclic graphs, generalized theta graphs, and graphs with $\leqslant 5$ vertices are all good.

In Section 6, we consider a strengthened variant of inequality (1.1) which in many instances is easier to prove than the original one. We say that a simple graph $G$ is extragood if for any doubly nonnegative function $g$ and any bounded measurable nonnegative functions $f_{1}, \ldots, f_{\mathrm{v}(G)}$ on $[0,1]$,

$$
\int_{[0,1]^{\mathrm{v}(G)}} \prod_{\left\{v_{i}, v_{j}\right\} \in E(G)} g\left(x_{i}, x_{j}\right) \prod_{i=1}^{\mathrm{v}(G)} f_{i}\left(x_{i}\right) d \mu^{\mathrm{v}(G)} \geqslant\left(\int_{[0,1]^{2}} f(x) g(x, y) f(y) d \mu^{2}\right)^{\mathrm{e}(G)}
$$


where $f(x)=\left(\prod_{i=1}^{\mathrm{v}(G)} f_{i}(x)\right)^{1 /(2 \mathrm{e}(G))}$. Obviously, if $G$ is extra-good, then $G$ is good. For each graph that we proved to be good, we also were able to prove that it is extra-good. It is possible that every good graph is extra-good.

A connection to Kohayakawa-Nagle-Rödl-Schacht conjecture is discussed in Section 8.

In Section 9, we discuss generalizations of inequality (1.1) for bounded measurable nonnegative symmetric functions of $r \geqslant 3$ variables.

\section{Doubly nonnegative and completely positive matrices}

A doubly nonnegative matrix is a real positive semidefinite square matrix with nonnegative entries. A completely positive matrix is a doubly nonnegative matrix which can be factorized as $A=B B^{T}$ where $B$ is a nonnegative (not necessarily square) matrix. It is well known (see [3]) that for any $k \geqslant 5$ there exist doubly nonnegative $k \times k$ matrices which are not completely positive.

For a $k \times k$ matrix $A=\left[a_{i j}\right]$, we define a function $g_{A}$ on $[0,1]^{2}$ as $g(x, y)=a_{i j}$ for $(i-1) / k<x \leqslant i / k,(j-1) / k<y \leqslant j / k$, and $g(x, y)=0$ if $x y=0$. Obviously, $g_{A}$ is a doubly nonnegative (completely positive) function if and only if $A$ is a doubly nonnegative (completely positive) matrix.

If $A(H)$ is the adjacency matrix of a $k$-vertex simple graph $H$, then the number of homomorphisms from $G$ to $H$ is equal to $t\left(G, g_{A(H)}\right) k^{\mathrm{v}(G)}$.

Notice that if a nonzero $k \times k$ matrix $A$ has zero diagonal, then $g_{A}$ is not nice, since $t\left(G, g_{A}\right)=0$ for any graph $G$ with chromatic number $\chi(G)>k$. We are going to demonstrate that presence of a single positive diagonal entry can be sufficient to make $g_{A}$ nice.

Theorem 2.1. Let $P$ be a symmetric permutation matrix of order $k$ with $a \geqslant 1$ diagonal entries equal to 1 , and $b \geqslant 1$ pairs of off-diagonal entries equal to $1(a+2 b=k)$. Then $g_{P}$, while not being positive semidefinite, is a nice function.

Proof. $P$ has eigenvalues 1 with multiplicity $a+b$, and -1 with multiplicity $b \geqslant 1$. Therefore, $P$ is not positive semidefinite. If graph $G$ has connected components $G_{1}, G_{2}, \ldots, G_{m}$ then $t(G, g)=\prod_{i=1}^{m} t\left(G_{i}, g\right)$. Hence, to prove (1.1) for $g=g_{P}$ it is sufficient to consider connected graphs $G$. If $G$ is a tree, then validity of (1.1) follows from (1.2) (Conjecture 1.1 has been proved for trees by various authors; a short proof can be found in [11]). Hence, we may assume that $G$ is not a tree. If $n=\mathrm{v}(G)$, then $\mathrm{e}(G) \geqslant n$. As $P$ has $a \geqslant 1$ diagonal entries equal to 1 , we get $t\left(G, g_{P}\right) \geqslant a(1 / k)^{n} \geqslant(1 / k)^{\mathrm{e}(G)}$ and $\int_{[0,1]^{2}} g_{P} d \mu^{2}=1 / k$.

\section{More on Conjecture 1.1}

The earliest known works where inequalities of type (1.1) and (1.2) appear are [18] and [2]. In 1959, Mulholland and Smith [18] proved that for any symmetric nonnegative matrix $A$ and any nonnegative vector $\mathbf{z}$,

$$
\left(\mathbf{z}^{\top} A^{k} \mathbf{z}\right) \cdot\left(\mathbf{z}^{\top} \mathbf{z}\right)^{k-1} \geqslant\left(\mathbf{z}^{\top} A \mathbf{z}\right)^{k}
$$


where equality takes place if and only if $\mathbf{z}$ is an eigenvector of $A$ or a zero vector. Note that (3.1) is a particular case of (1.1) where $H$ is the $k$-edge path $P_{k}$.

Almost at the same time, Atkinson, Watterson and Moran [2] proved that $\mathrm{nm}$. $s\left(A A^{\top} A\right) \geqslant s(A)^{3}$, where $A$ is an (asymmetric) nonnegative $(n \times m)$-matrix, and $s(A)$ is the sum of entries of $A$. They presented their inequality in both matrix and integral form, and conjectured validity of (1.2) for $H=P_{k}$ with $k \geqslant 3$.

In 1965, Blakley and Roy [4], being unaware of the article [18], rediscovered (3.1).

Lately, Conjecture 1.1 has been proved for various bipartite graphs (see [6, 7, 8, 9, 12, $15,17,19,21,22,23,24,25])$, among them: trees, complete bipartite graphs, and graphs with 9 vertices or less. Some of the authors restricted (1.2) to symmetric functions $h$. Nevertheless, the proofs of their results can be extended to asymmetric $h$ as well. Let $\mathfrak{S}$ be the class of bipartite graphs that satisfy Conjecture 1.1 , and $\mathfrak{S}_{*}$ be the class of bipartite graphs $H$ that satisfy (1.2) for all $h \in \mathcal{G}_{+}$. Obviously, $\mathfrak{S} \subseteq \mathfrak{S}_{*}$. It would be nice to prove $\mathfrak{S}=\mathfrak{S}_{*}$.

Theorem 3.1. If $H \in \mathfrak{S}_{*}$ is symmetric, then $H \in \mathfrak{S}$.

In the proof of Theorem 3.1, we will use the so called "tensor-trick" lemma.

Lemma $3.2([21,22])$. If there exists a constant $c=c_{H}>0$ such that for any $h \in \mathcal{H}_{+}$, $t(H, h) \geqslant c \cdot\left(\int_{[0,1]^{2}} h d \mu^{2}\right)^{\mathrm{e}(H)}$, then $H \in \mathfrak{S}$.

Proof of Theorem 3.1. It is sufficient to consider the case when $H$ is connected. Denote by $n$ the size of each vertex set of $H$, so the total number of vertices is $2 n$. Let $h \in \mathcal{H}_{+}$. Define its "transpose" $h^{\top}$ as $h^{\top}(x, y)=h(y, x)$. As $H$ is symmetric, $t(H, h)=t\left(H, h^{\top}\right)$. Define symmetric function $\tilde{h} \in \mathcal{G}_{+}$as follows:

$$
\tilde{h}(x, y)= \begin{cases}0 & \text { if } 0 \leqslant x, y<1 / 2 \\ h(2 x, 2 y-1) & \text { if } 0 \leqslant x<1 / 2 \leqslant y \leqslant 1 \\ h(2 y, 2 x-1) & \text { if } 0 \leqslant y<1 / 2 \leqslant x \leqslant 1 \\ 0 & \text { if } 1 / 2 \leqslant x, y \leqslant 1\end{cases}
$$

Notice that $\int_{[0,1]^{2}} \tilde{h} d \mu^{2}=(1 / 2) \int h_{[0,1]^{2}} d \mu^{2}$. As $H$ is connected,

$$
t(H, \tilde{h})=2^{-2 n}\left(t(H, h)+t\left(H, h^{\top}\right)\right)=2^{1-2 n} t(H, h) .
$$

Since $H \in \mathfrak{S}_{*}$, we get $t(H, \tilde{h}) \geqslant\left(\int_{[0,1]^{2}} \tilde{h} d \mu^{2}\right)^{\mathrm{e}(H)}$. Hence,

$$
t(H, h) \geqslant 2^{2 n-1-\mathrm{e}(H)}\left(\int_{[0,1]^{2}} h d \mu^{2}\right)^{\mathrm{e}(H)}
$$

and by Lemma 3.2, $H \in \mathfrak{S}$. 
Remark 3.3. It is a classical fact that there exists a measure preserving bijection between any two atomless measure spaces with total measure 1 . In particular, if $\mu_{1}$ and $\mu_{2}$ are atomless measures on $[0,1]$, and a bipartite graph $H \in \mathfrak{S}$ has vertex sets of sizes $n$ and $m$, then for any bounded non-negative function $h$ on $[0,1]^{2}$, measurable with respect to $\mu_{1} \otimes \mu_{2}$,

$$
\int_{[0,1]^{n+m}} \prod_{\left(v_{i}, w_{j}\right) \in E(H)} h\left(x_{i}, y_{j}\right) d \mu_{1}^{n} d \mu_{2}^{m} \geqslant\left(\int_{[0,1]^{2}} h d \mu_{1} d \mu_{2}\right)^{\mathrm{e}(H)} .
$$

Theorem 3.4. If Conjecture 1.1 holds for a bipartite graph $H$, and all vertices from the first vertex set of $H$ have the same degree $a$, then $t(H, h) \geqslant t\left(K_{1, a}, h\right)^{\mathrm{e}(H) / a}$ for any $h \in \mathcal{H}_{+}$. If all vertices from the second vertex set of $H$ have the same degree $b$, then $t(H, h) \geqslant t\left(K_{b, 1}, h\right)^{\mathrm{e}(H) / b}$ for any $h \in \mathcal{H}_{+}$.

Proof. We will prove the first part of the statement (the proof of the second part is similar). Notice that $\mathrm{e}(H)=n a$. It is sufficient to consider functions $h \in \mathcal{H}_{+}$that are separated from zero: $\inf _{[0,1]^{2}} h>0$. Denote $\varphi(x)=\int_{[0,1]} h(x, y) d \mu(y)$. Then $c=$ $\int_{[0,1]} \varphi(x)^{a} d \mu>0$, and $f(x)=\varphi(x)^{a} / c$ is positive and bounded on $[0,1]$. Consider a measure $\mu_{*}$ on $[0,1]$ defined by $d \mu_{*}=f d \mu$, so $\mu_{*}([0,1])=1$. Denote $\widehat{h}(x, y)=h(x, y) f(x)^{-1 / a}$. Clearly, $\widehat{h}$ is bounded and measurable with respect to $\mu_{*} \otimes \mu$. By Remark 3.3,

$$
\begin{aligned}
t(H, h)^{1 / n} & =\left(\int_{[0,1]^{n+m}} \prod_{\left(v_{i}, w_{j}\right) \in E(H)} h\left(x_{i}, y_{j}\right) d \mu^{n} d \mu^{m}\right)^{1 / n} \\
& =\left(\int_{[0,1]^{n+m}} \prod_{\left(v_{i}, w_{j}\right) \in E(H)} \widehat{h}\left(x_{i}, y_{j}\right) d \mu_{*}^{n} d \mu^{m}\right)^{1 / n} \\
& \geqslant\left(\int_{[0,1]^{2}} \widehat{h}(x, y) d \mu_{*}(x) d \mu(y)\right)^{a} \\
& =\left(\int_{[0,1]^{2}} h(x, y) f(x)^{-1 / a} d \mu_{*}(x) d \mu(y)\right)^{a} \\
& =\left(\int_{[0,1]^{2}} h(x, y) f(x)^{1-(1 / a)} d \mu^{2}\right)^{a} \\
& \left.=\left(\int_{[0,1]} \varphi(x) f(x)^{1-(1 / a)} d \mu\right)^{a}\right)_{[0,1]} \varphi(x)^{a} d \mu=t\left(K_{1, a}, h\right) .
\end{aligned}
$$

Theorem 3.5. For a fixed graph $G$, inequality (1.1) holds for any completely positive function $g$ if and only if Conjecture 1.1 holds for $H=\operatorname{Sub}(G)$.

Proof. Suppose that Conjecture 1.1 holds for $H=\operatorname{Sub}(G)$, and a function $g$ is completely positive. There exists $h \in \mathcal{H}_{+}$such that $g(x, y)=\int_{[0,1]} h(x, z) h(y, z) d \mu(z)$. Then $t(G, g)=$ 
$t(H, h)$. Every vertex in the second vertex set of $H$ has degree 2. By Theorem 3.4, we have $t(H, h) \geqslant t\left(K_{2,1}, h\right)^{\mathrm{e}(H) / 2}$. As $\mathrm{e}(G)=\mathrm{e}(H) / 2$ and $t\left(K_{2,1}, h\right)=\int_{[0,1]^{2}} g d \mu^{2}$, we get (1.1).

Now suppose that (1.1) holds for any completely positive function $g$. Let $h \in \mathcal{H}_{+}$. Set $g(x, y)=\int_{[0,1]} h(x, z) h(y, z) d \mu(z)$. Then $t(H, h)=t(G, g) \geqslant\left(\int_{[0,1]^{2}} g d \mu^{2}\right)^{\mathrm{e}(G)} \geqslant$ $\left(\int_{[0,1]^{2}} h d \mu^{2}\right)^{2 \mathrm{e}(G)}=\left(\int_{[0,1]^{2}} h d \mu^{2}\right)^{\mathrm{e}(H)}$.

\section{Subdivisions that are norming}

We say that a bipartite graph $H$ with vertex sets $V=\left\{v_{1}, v_{2}, \ldots, v_{n}\right\}$ and $W=\left\{w_{1}, w_{2}\right.$, $\left.\ldots, w_{m}\right\}$ has the Hölder property if for any assignment $f: E(H) \rightarrow \mathcal{H}$,

$$
\left(\int_{[0,1]^{n+m}} \prod_{e=\left(v_{i}, w_{j}\right) \in E(H)} f_{e}\left(x_{i}, y_{j}\right) d \mu^{n+m}\right)^{\mathrm{e}(H)} \leqslant \prod_{e \in E(H)} t\left(H, f_{e}\right) .
$$

It is known (see $[10,16]$ ) that every graph $H$ with the Hölder property (except a star with even number of edges) is a norming graph: $t(H, h)^{1 / \mathrm{e}(H)}$ is a norm on $\mathcal{H}$. Conversely, every norming graph has the Hölder property.

Theorem 4.1. If $\operatorname{Sub}(G)$ has the Hölder property then $G$ is good.

Proof. Let $H=\operatorname{Sub}(G)$. If $g(x, y)=\int_{[0,1]} h(x, z) h(y, z) d \mu(z)$ then $t(G, g)=t(H, h)$. Select a pair of edges $e^{\prime}, e^{\prime \prime}$ in $H$ which subdivide the same edge of $G$. Assign $f_{e^{\prime}}=f_{e^{\prime \prime}}=h$ and $f_{e}=1$ for $e \neq e^{\prime}, e^{\prime \prime}$. Then the left hand side of $(4.1)$ is $\left(\int_{[0,1]^{2}} g d \mu^{2}\right)^{2 \mathrm{e}(G)}$, and the right hand side is $t(G, g)^{2}$.

The 1-subdivision of cycle $C_{n}$ is an even cycle $C_{2 n}$ which is a norming graph. The 1 -subdivision of the octahedron $K_{2,2,2}$ is norming (see [8, Example 4.15]). Hence, $C_{n}$ and $K_{2,2,2}$ are good graphs.

In a norming graph, the degrees of vertices are even (see Observation 2.5 in [10]). Hence, $\operatorname{Sub}\left(K_{2 r}\right)$ is not norming. While $\operatorname{Sub}\left(K_{3}\right)=C_{6}$ is norming, it is not known whether $\operatorname{Sub}\left(K_{2 r+1}\right)$ with $r \geqslant 2$ is norming. We will prove in the next section that all complete graphs are good.

\section{Complete graphs are good}

Theorem 5.1. If graph $G$ is good, and graph $G_{1}$ is obtained from $G$ by adding a new vertex adjacent to all vertices of $G$, then $G_{1}$ is good.

Corollary 5.2. The complete graphs are good.

Theorem 5.3. If graph $G$ is good, and graph $G_{2}$ is obtained from $G$ by adding two vertices adjacent to all vertices of $G$ but not to each other, then $G_{2}$ is good. 
Corollary 5.4. Any graph whose complement is a set of independent edges is good.

To prove Theorems 5.1 and 5.3 we need a couple of auxiliary results. For $g \in \mathcal{G}_{+}$, we denote $\|g\|=\int_{[0,1]^{2}} g d \mu^{2}$.

Lemma 5.5. If function $g$ is doubly nonnegative, then $t\left(K_{3}, g\right) \geqslant t\left(K_{1,2}, g\right)^{3 / 2}$ and $t\left(K_{4}-e, g\right) \geqslant t\left(K_{2,2}, g\right)^{5 / 4}$.

Proof. As $g$ is doubly nonnegative, $g(x, y)=\int_{[0,1]} h(x, z) h(y, z) d \mu(z)$. Then $t\left(K_{3}, g\right)=$ $t\left(C_{6}, h\right), t\left(K_{1,2}, g\right)=t\left(P_{4}, h\right), t\left(K_{2,2}, g\right)=t\left(C_{8}, h\right)$, where $P_{4}$ denotes the 4-edge path.

As $C_{6}$ is norming, and $P_{4}$ is a subgraph of $C_{6}$, we have $t\left(C_{6}, h\right)^{1 / 6} \geqslant t\left(P_{4}, h\right)^{1 / 4}$.

By the Cauchy-Schwarz inequality, $t\left(K_{4}-e, g\right)\|g\| \geqslant t\left(K_{3}, g\right)^{2}=t\left(C_{6}, h\right)^{2}$. As $t\left(C_{2 k}, h\right)^{1 / 2 k}$ is the $(2 k)$-th Schatten norm of $h$, we get $t\left(C_{6}, h\right)^{1 / 6} \geqslant t\left(C_{8}, h\right)^{1 / 8}$. Hence, $t\left(K_{4}-e, g\right) \geqslant t\left(K_{2,2}, g\right)^{3 / 2}\|g\|^{-1} \geqslant t\left(K_{2,2}, g\right)^{5 / 4}$.

For $n>m \geqslant 1$, let $K_{n}-K_{m}$ denote the complement of $K_{m}$ in $K_{n}$. It follows from Lemma 5.5 that for $m=1,2$ and any doubly nonnegative function $g$,

$$
t\left(K_{m+2}-K_{m}, g\right) \geqslant t\left(K_{2, m}, g\right) \cdot\|g\| .
$$

Thus, Theorems 5.1 and 5.3 follow from the next proposition.

Proposition 5.6. Let integer $m$ be such that (5.1) holds for all doubly nonnegative functions. If graph $G$ is good, and graph $G_{m}$ is obtained from $G$ by adding a group of $m$ independent vertices that are adjacent to all vertices of $G$, then $G_{m}$ is good.

Proof. We may assume $\mathrm{v}(G) \geqslant 2$. It is sufficient to consider functions $g$ that are separated from zero: $\inf _{[0,1]^{2}} g>0$. Then function $\varphi\left(x_{1}, \ldots, x_{m}\right)=\int_{[0,1]} \prod_{i=1}^{m} g\left(x_{i}, y\right) d \mu(y)$ is positive and bounded on $[0,1]^{m}$. For each $\mathbf{x}=\left(x_{1}, \ldots, x_{m}\right) \in[0,1]^{m}$, consider measure $\mu_{\mathbf{x}}$ on $[0,1]$, defined by $d \mu_{\mathbf{x}}=f_{\mathbf{x}} d \mu$, where $f_{\mathbf{x}}(y)=\prod_{i=1}^{m} g\left(x_{i}, y\right) \varphi\left(x_{1}, \ldots, x_{m}\right)^{-1}$. It is easy to see that $\mu_{\mathbf{x}}([0,1])=1$, and $g$ is bounded and measurable with respect to $\mu_{\mathbf{x}} \otimes \mu_{\mathbf{x}}$. By Remark 3.3,

$$
t\left(G, g, \mu_{\mathbf{x}}\right) \stackrel{\text { def }}{=} \int_{[0,1]^{\mathrm{v}(G)}} \prod_{\left\{v_{i}, v_{j}\right\} \in E(G)} g\left(y_{i}, y_{j}\right) d \mu_{\mathbf{x}}^{\mathrm{v}(G)} \geqslant\left(\int_{[0,1]^{2}} g d \mu_{\mathbf{x}}^{2}\right)^{\mathrm{e}(G)} .
$$

Hence,

$$
\begin{aligned}
& t\left(G_{m}, g\right)=\int_{[0,1]^{m}} t\left(G, g, \mu_{x_{1}, \ldots, x_{m}}\right) \varphi\left(x_{1}, \ldots, x_{m}\right)^{\mathrm{v}(G)} d \mu\left(x_{1}\right) \cdots d \mu\left(x_{m}\right) \\
& \geqslant \int_{[0,1]^{m}}\left(\int_{[0,1]^{2}} g d \mu_{x_{1}, \ldots, x_{m}}^{2}\right)^{\mathrm{e}(G)} \varphi\left(x_{1}, \ldots, x_{m}\right)^{\mathrm{v}(G)} d \mu\left(x_{1}\right) \cdots d \mu\left(x_{m}\right) \\
&=\int_{[0,1]^{m}}\left(\int_{[0,1]^{2}} g(y, z) \prod_{i=1}^{m}\left(g\left(x_{i}, y\right) g\left(x_{i}, z\right)\right) d \mu(y) d \mu(z)\right)^{\mathrm{e}(G)} \\
& \times \varphi\left(x_{1}, \ldots, x_{m}\right)^{\mathrm{v}(G)-2 \mathrm{e}(G)} d \mu\left(x_{1}\right) \cdots d \mu\left(x_{m}\right) .
\end{aligned}
$$


As $1+\mathrm{e}(G)-\mathrm{v}(G) / 2 \leqslant \mathrm{e}(G)$, by using the Hölder inequality, we get

$$
\begin{aligned}
t\left(G_{m}, g\right) \cdot t & \left(K_{2, m}, g\right)^{\mathrm{e}(G)-\mathrm{v}(G) / 2} \\
& =t\left(G_{m}, g\right) \cdot\left(\int_{[0,1]^{m}} \varphi^{2}\left(x_{1}, \ldots, x_{m}\right) d \mu\left(x_{1}\right) \cdots d \mu\left(x_{m}\right)\right)^{\mathrm{e}(G)-\mathrm{v}(G) / 2} \\
& \geqslant\left(\int_{[0,1]^{m+2}} g(y, z) \prod_{i=1}^{m}\left(g\left(x_{i}, y\right) g\left(x_{i}, z\right)\right) d \mu(y) d \mu(z) d \mu\left(x_{1}\right) \cdots d \mu\left(x_{m}\right)\right)^{\mathrm{e}(G)} \\
& =t\left(K_{m+2}-K_{m}, g\right)^{\mathrm{e}(G)}
\end{aligned}
$$

As Conjecture 1.1 holds for complete bipartite graphs (see [21]), $K_{2, m}$ is good. By using (5.1), we get

$$
\begin{aligned}
t\left(G_{m}, g\right) & \geqslant t\left(K_{2, m}, g\right)^{-\mathrm{e}(G)+\mathrm{v}(G) / 2} t\left(K_{2, m}, g\right)^{\mathrm{e}(G)}\|g\|^{\mathrm{e}(G)} \\
& =t\left(K_{2, m}, g\right)^{\mathrm{v}(G) / 2}\|g\|^{\mathrm{e}(G)} \geqslant\|g\|^{m \mathrm{v}(G)}\|g\|^{\mathrm{e}(G)}=\|g\|^{\mathrm{e}\left(G_{m}\right)}
\end{aligned}
$$

\section{Extra-good graphs}

Theorem 6.1. If $G$ is vertex-transitive and good, then $G$ is extra-good.

Proof. We denote $n=\mathrm{v}(G), f(x)=\left(\prod_{i=1}^{n} f_{i}(x)\right)^{1 /(2 \mathrm{e}(G))}$ and $\tilde{g}(x, y)=f(x) g(x, y) f(y)$. Notice that $\tilde{g}$ is doubly nonnegative. Since $G$ is vertex-transitive, any permutation of the functions $f_{1}, f_{2}, \ldots, f_{n}$ does not change the value of the integral on the left hand side of (1.3). By applying the Hölder inequality to the geometric mean of all $n$ ! possible integrals, we get

$$
\begin{aligned}
\int_{[0,1]^{n}} \prod_{\left\{v_{i}, v_{j}\right\} \in E(G)} g\left(x_{i}, x_{j}\right) \prod_{i=1}^{n} f_{i}\left(x_{i}\right) d \mu^{n} & \geqslant \int_{[0,1]^{n}} \prod_{\left\{v_{i}, v_{j}\right\} \in E(G)} g\left(x_{i}, x_{j}\right) \prod_{i=1}^{n} f\left(x_{i}\right)^{2 \mathrm{e}(G) / n} d \mu^{n} \\
& =\int_{[0,1]^{n}} \prod_{\left\{v_{i}, v_{j}\right\} \in E(G)} \tilde{g}\left(x_{i}, x_{j}\right) d \mu^{n} .
\end{aligned}
$$

Since $G$ is good,

$$
\int_{[0,1]^{n}} \prod_{\left\{v_{i}, v_{j}\right\} \in E(G)} \tilde{g}\left(x_{i}, x_{j}\right) d \mu^{n} \geqslant\left(\int_{[0,1]^{2}} \tilde{g}(x, y) d \mu^{2}\right)^{\mathrm{e}(G)} .
$$

Corollary 6.2. Cycles and complete graphs are extra-good.

Theorem 6.3. If vertex $v$ is a leaf in graph $G$, and $G-v$ is extra-good, then $G$ is extra-good too. 
Proof. Let $\mathrm{v}(G)=n+1, V(G)=\left\{v_{1}, \ldots, v_{n}, v_{n+1}\right\}$, and $v=v_{n+1}$ is a leaf. For a set of functions $f_{1}, \ldots, f_{n}, f_{n+1}$, define $\tilde{f}_{i}=f_{i}$ for $i=1, \ldots, n-1$, and $\tilde{f}_{n}(x)=$ $f_{n}(x) \int_{[0,1]} g(x, y) f_{n+1}(y) d \mu(y)$. Then

$$
\begin{aligned}
\int_{[0,1]^{n+1}} \prod_{\left\{v_{i}, v_{j}\right\} \in E(G)} g\left(x_{i}, x_{j}\right) \prod_{i=1}^{n+1} f_{i}\left(x_{i}\right) d \mu^{n+1} & =\int_{[0,1]^{n}} \prod_{\left\{v_{i}, v_{j}\right\} \in E(G-v)} g\left(x_{i}, x_{j}\right) \prod_{i=1}^{n} \tilde{f}_{i}\left(x_{i}\right) d \mu^{n} \\
& \geqslant\left(\int_{[0,1]^{2}} \tilde{f}(x) g(x, y) \tilde{f}(y) d \mu^{2}\right)^{\mathrm{e}(G)-1} \\
& =I_{0}^{\mathrm{e}(G)-1},
\end{aligned}
$$

where $\tilde{f}(x)=\left(\prod_{i=1}^{n} \tilde{f}_{i}(x)\right)^{1 /(2 \mathrm{e}(G)-2)}$. Set $\varphi(x)=\int_{[0,1]} g(x, y) f_{n+1}(y) d \mu(y)$,

$$
I_{1}=\int_{[0,1]^{2}} \varphi(x)^{-1} g(x, y) f_{n+1}(y) d \mu^{2}, \quad I_{2}=\int_{[0,1]^{2}} f_{n+1}(x) g(x, y) \varphi(y)^{-1} d \mu^{2} .
$$

It is easy to see that $I_{1}=\int_{[0,1]} \varphi(x)^{-1} \varphi(x) d \mu=1$, and similarly, $I_{2}=1$. By the Hölder inequality,

$$
I_{0}^{\mathrm{e}(G)-1} \cdot 1 \cdot 1=I_{0}^{\mathrm{e}(G)-1} \cdot I_{1}^{1 / 2} \cdot I_{2}^{1 / 2} \geqslant\left(\int_{[0,1]^{2}} f(x) g(x, y) f(x) d \mu^{2}\right)^{\mathrm{e}(G)},
$$

where

$$
f^{2 \mathrm{e}(G)}=(\tilde{f})^{2 \mathrm{e}(G)-2} \varphi^{-1} f_{n+1}=\prod_{i=1}^{n} \tilde{f}_{i} \cdot \varphi^{-1} f_{n+1}=\prod_{i=1}^{n-1} f_{i} \cdot\left(f_{n} \varphi\right) \cdot \varphi^{-1} f_{n+1}=\prod_{i=1}^{n+1} f_{i} .
$$

A connected graph $G$ is called unicyclic if $\mathrm{e}(G)=\mathrm{v}(G)$, and bicyclic if $\mathrm{e}(G)=\mathrm{v}(G)+1$.

Corollary 6.4. Trees and unicyclic graphs are extra-good.

Let $P\left(k_{1}, k_{2}, \ldots, k_{r}\right)$ denote a graph consisting of two vertices joined by $r$ internally disjoint paths of length $k_{1}, k_{2}, \ldots, k_{r}$. Such a graph is called theta graph (see [5]). If $r=2$, then $P\left(k_{1}, k_{2}\right)$ is simply a cycle of length $k_{1}+k_{2}$.

Theorem 6.5. $P\left(k_{1}, k_{2}, \ldots, k_{r}\right)$ is extra-good.

Let $C\left(k_{1}, k_{2}, m\right)$ denote a graph which consists of two cycles of length $k_{1}$ and $k_{2}$ connected by a path of length $m \geqslant 0$ (when $m=0$, the cycles share a vertex). Notice that $P\left(k_{1}, k_{2}, k_{3}\right)$ and $C\left(k_{1}, k_{2}, m\right)$ are the only bicyclic graphs without leaves. In view of Theorems 6.3 and 6.5, in order to prove that all bicyclic graphs are extra-good, it is sufficient to show that $C\left(k_{1}, k_{2}, m\right)$ is extra-good. This will follow from the next result.

Theorem 6.6. Let graph $G_{0}$ be formed by attaching a cycle to one of the vertices of graph $G$. If $G$ is extra-good, then $G_{0}$ is extra-good. 
Corollary 6.7. Bicyclic graphs are extra-good.

The proofs of Theorems 6.5 and 6.6 are given in the appendix.

Consider a tree $T$ whose vertices are arbitrarily colored in black and white so that at least one vertex is black. Take $r$ disjoint copies of $T$ and glue together "sister" black vertices from different copies. We call the resulting graph a multitree. For example $P(k, k, \ldots, k)$ is a multitree. The case of even cycle in Theorem 6.6 is a particular case of the following statement.

Theorem 6.8. Let graph $G_{0}$ be formed by gluing a black vertex of a multitree to one of the vertices of graph $G$. If $G$ is extra-good, then $G_{0}$ is extra-good.

Theorem 6.9. If graph $G$ is extra-good, and graph $G_{1}$ is obtained from $G$ by adding a new vertex adjacent to all vertices of $G$, then $G_{1}$ is extra-good.

Theorem 6.10. If graph $G$ is extra-good, and graph $G_{2}$ is obtained from $G$ by adding two vertices adjacent to all vertices of $G$ but not to each other, then $G_{2}$ is extra-good.

Theorems 6.8, 6.9, and 6.10 are not used in the rest of the article. We omit their proofs as they are very similar to the proofs of Theorems 6.6, 5.1, and 5.3.

\section{Graphs with small number of vertices}

Theorem 7.1. All graphs with 5 vertices or less are good.

Proof. If graph $G$ has connected components $G_{1}, G_{2}, \ldots, G_{m}$ then $t(G, g)=\prod_{i=1}^{m} t\left(G_{i}, g\right)$. Hence, it is sufficient to consider connected graphs $G$ only. As Conjecture 1.1 has been proved for bipartite graphs with 9 vertices or less, it is sufficient to consider connected simple graphs with at least one odd cycle. The results of Sections 5 and 6 cover all such graphs with $\leqslant 5$ vertices. A table of all 5 -vertex graphs that do not have isolated vertices can be found in [1, Figure 6].

Some 6 -vertex graphs are not covered by the results of Sections 5 and 6 , but for almost all of them we were able to prove that they are good by using the Cauchy-Schwarz and Hölder inequalities. The only graph with 6 vertices that we were unable to prove to be good is the complement of the 5-edge path.

\section{Locally dense graphs}

A simple graph $H$ is called $(\varepsilon, d)$-dense if every subset $X \subseteq V(H)$ of size $|X| \geqslant \varepsilon|V(H)|$ spans at least $\frac{d}{2}|X|^{2}$ edges.

Conjecture 8.1 ([13]). For any graph $G$ and $\delta, d \in(0,1)$, there exists $\varepsilon=\varepsilon(\delta, d, G)$ such that there are at least $\left(d^{\mathrm{e}(G)}-\delta\right) \mathrm{v}(H)^{\mathrm{v}(G)}$ homomorphisms of $G$ into any sufficiently large $(\varepsilon, d)$-dense graph $H$. 
When $\chi(G)=2$, Conjecture 8.1 follows from Conjecture 1.1. It is known that Conjecture 8.1 holds for complete graphs and multipartite complete graphs (see[13, 20]). Christian Reiher [20] proved Conjecture 8.1 for odd cycles. Joonkyung Lee [14] proved that adding an edge to a cycle or a tree produces graphs that satisfy the conjecture. He also proved that Conjecture 8.1 holds for a class of graphs obtained by gluing complete multipartite graphs (or odd cycles) in a tree-like way. All graphs with 5 vertices or less satisfy the conjecture.

For a nonnegative symmetric $k \times k$ matrix $A$, we define its density $d(A)$ as the minimum of $\mathbf{x}^{\top} A \mathbf{x}$ over all nonegative $k$-dimensional vectors $\mathbf{x}$ with the sum of entries equal to 1 . Clearly, $d(A) \leqslant\left\|g_{A}\right\|$. We call a graph $G$ density-friendly if $t\left(G, g_{A}\right) \geqslant d(A)^{\mathrm{e}(G)}$ for any $A$. It is easy to see that any density-friendly graph satisfies Conjecture 8.1, but the converse is not obvious.

While Conjectures 1.2 and 8.1 looks very different, the sets of graphs which are known to satisfy them are surprisingly similar.

One can try to build a bridge between these two topics by defining

$$
c(A)=\inf _{G} t\left(G, g_{A}\right)^{1 / \mathrm{e}(G)} .
$$

Then Conjecture 1.2 claims that $c(A)=\left\|g_{A}\right\|$ for any doubly nonnegative matrix $A$, and Conjecture 8.1 claims that $c(A) \geqslant d(A)$ for any nonnegative symmetric matrix $A$.

\section{$9 \quad$ Functions of $r$ variables}

Let $G$ be an $r$-uniform hypergraph with vertex set $V(G)=\left\{v_{1}, v_{2}, \ldots, v_{n}\right\}$ and edge set $E(G)$ (edges are $r$-element subsets of the vertex set). Let $g$ be a bounded symmetric measurable nonnegative function defined on $[0,1]^{r}$. Denote $\|g\|=\int_{[0,1]^{r}} g d \mu^{r}$ and

$$
t(G, g)=\int_{[0,1]^{n}} \prod_{\left\{v_{i_{1}}, v_{i_{2}}, \ldots, v_{i_{r}}\right\} \in E(G)} g\left(x_{i_{1}}, x_{i_{2}}, \ldots, x_{i_{r}}\right) d \mu^{n} .
$$

Problem 9.1. Characterize functions $g$ such that

$$
t(G, g) \geqslant\|g\|^{|E(G)|}
$$

holds for every $r$-uniform hypergraph $G$.

When $r=1$, it is obvious that (9.1) holds for any nonnegative function $h$ on $[0,1]$.

The incidence graph of an $r$-uniform hypergraph $G$ is a bipartite graph $\operatorname{Inc}(G)$ with vertex sets $V(G)$ and $E(G)$, where $v \in V(G)$ and $e \in E(G)$ form an edge $\{v, e\}$ in $\operatorname{Inc}(G)$ if and only if $v \in e$ in $G$.

If there is a function $h \in \mathcal{H}$ such that

$$
g\left(x_{1}, x_{2}, \ldots, x_{r}\right)=\int_{[0,1]} \prod_{i=1}^{r} h\left(x_{i}, y\right) d \mu(y)
$$


then $t(G, g)=t(\operatorname{Inc}(G), h)$.

Similarly to Theorem 3.5, if $\operatorname{Inc}(G)$ satisfies Conjecture 1.1, then (9.1) holds for functions $g$ that have representation (9.2) with nonnegative $h$. Similarly to Theorem 4.1, if $\operatorname{Inc}(G)$ is norming (it requires $r$ to be even), then (9.1) holds for functions $g$ that have representation (9.2), where $h \in \mathcal{H}$ can take negative values.

\section{Acknowledgements}

The author would like to thank Joonkyung Lee for his valuable comments and suggestions.

\section{References}

[1] P. Adams, D. Bryant, and M. Buchanan. A survey on the existence of $G$-designs. J. Combin. Designs, 16(5):373-410, 2008. https://doi.org/10.1002/jcd. 20170.

[2] F. V. Atkinson, G. A. Watterson, and P. A. D. Moran. A matrix inequality. Quarterly J. of Math., 11(42):137-140, 1960. https://doi.org/10.1093/qmath/11.1.137.

[3] A. Berman and N. Shaked-Monderer. Completely Positive Matrices, World Scientific, 2003. https://doi.org/10.1142/5273.

[4] G. R. Blakley and P. Roy. Hölder type inequality for symmetric matrices with nonnegative entries. Proc. Amer. Math. Soc., 16(6):1244-1245, 1965. https://doi .org/10.1090/S0002-9939-1965-0184950-9.

[5] A. Blinco. Theta graphs, graph decompositions and related graph labelling techniques. Bull. Austral. Math. Soc., 69(1):173-175, 2004. https://doi.org/10.1017/S0004972700034377.

[6] D. Conlon, J. Fox, and B. Sudakov. An approximate version of Sidorenko's conjecture. Geom. Funct. Anal., 20(6):1354-1366, 2010. https://doi.org/10.1007/s00039-010-0097-0.

[7] D. Conlon, J. H. Kim, C. Lee, and J. Lee. Some advances on Sidorenko's conjecture. J. London Math. Soc., 98(3):593-608, 2018. https://doi.org/10.1112/jlms. 12142.

[8] D. Conlon and J. Lee. Finite reflection groups and graph norms. Adv. Math., 315:130165, 2017. https://doi.org/10.1016/j.aim.2017.05.009.

[9] D. Conlon and J. Lee. Sidorenko's conjecture for blow-ups. Discrete Analysis, to appear. arXiv:1809.01259, 2018.

[10] H. Hatami. Graph norms and Sidorenko's conjecture. Israel J. Math., 175(1):125150, 2010. https://doi .org/10.1007/s11856-010-0005-1.

[11] C. Jagger, P. Šťviček, and A. Thomason. Multiplicities of subgraphs. Combinatorica, 16(1):123-141, 1996. https://doi.org/10.1007/BF01300130.

[12] J. H. Kim, C. Lee, and J. Lee. Two approaches to Sidorenko's conjecture. Trans. Amer. Math. Soc., 368(7):5057-5074, 2016. https://doi.org/10.1090/tran/6487. 
[13] Y. Kohayakawa, B. Nagle, V. Rödl, and M. Schacht. Weak hypergraph regularity and linear hypergraphs. J. Combin. Theory Ser. B, 100(2):151-160, 2010. https://doi.org/10.1016/j.jctb.2009.05.005.

[14] J. Lee. On some graph densities in locally dense graphs. Random Struct Alg., 58:322344, 2021. https://doi.org/10.1002/rsa. 20974.

[15] J. L. X. Li and B. Szegedy. On the logarithmic calculus and Sidorenko's conjecture. arXiv:1107.1153, 2011.

[16] L. Lovász. Large networks and graph limits, volume 60 of Colloquium Publications. AMS, 2012. https://doi.org/10.1090/coll/060.

[17] L. Lovász. Subgraph densities in signed graphons and the local Sidorenko conjecture. Electr. J. Combin., 18(1) \#P127, 2011.

[18] H. P. Mulholland and C. A. B. Smith. An inequality arising in genetical theory. Amer. Math. Monthly, 66(8):673-683, 1959. https://doi .org/10.2307/2309342.

[19] O. Parczyk. On Sidorenko's conjecture, Master's thesis, Freie Universität Berlin, 2014. http://www. uni-frankfurt.de/58522166.

[20] C. Reiher. Counting odd cycles in locally dense graphs. J. Combin. Theory Ser. B, 105:1-5, 2014. https://doi.org/10.1016/j.jctb.2013.12.002.

[21] A. Sidorenko. Inequalities for functionals generated by bipartite graphs. Discrete Math. Appl., 2(5):489-504, 1992. https://doi.org/10.1515/dma.1992 .2 .5.489.

[22] A. Sidorenko. A correlation inequality for bipartite graphs. Graphs and Combinatorics, 9(2):201-204, 1993. https://doi.org/10.1007/BF02988307.

[23] A. Sidorenko. An analytic approach to extremal problems for graphs and hypergraphs. In Extremal Problems for Finite Sets (Visegrád, 1991), volume 3 of Bolyai Soc. Math. Stud., pages 423-455. János Bolyai Math. Soc., Budapest, 1994.

[24] B. Szegedy. An information theoretic approach to Sidorenko's conjecture. arXiv:1406.6738, 2014.

[25] B. Szegedy. Sparse graph limits, entropy maximization and transitive graphs. arXiv:1504.00858, 2015.

\section{A Proofs of Theorems 6.5 and 6.6}

Proof of Theorem 6.5. The proof would be easy if all $k_{i}$ were even: then we could use the Cauchy-Schwarz inequality and the fact that the tree formed by paths of length $k_{1} / 2, k_{2} / 2, \ldots, k_{r} / 2$ connected at one endpoint is an extra-good graph. To deal with odd values of $k_{i}$, we are going to subdivide the middle edge of the path. We assume that $k_{1}, \ldots, k_{s}$ are odd, and $k_{s+1}, \ldots, k_{r}$ are even $(0 \leqslant s \leqslant r)$. We denote the vertices of the $i$ th path by $v_{i 0}, v_{i 1}, \ldots, v_{i k_{i}}$. Gluing together vertices $v_{i 0}$ for all $i$ produces vertex $v_{0}$. Gluing together vertices $v_{i k_{i}}$ produces vertex $v_{\infty}$. The total number of vertices is $n=2+\sum_{i=1}^{r}\left(k_{i}-1\right)$, and the number of edges is $m=\sum_{i=1}^{r} k_{i}$. 
Let $g$ be a doubly negative function: $g(x, y)=\int_{[0,1]} h(x, z) h(y, z) d \mu(z)$. Let $f_{0}, f_{\infty}, f_{i j}$ $\left(1 \leqslant i \leqslant r, 1 \leqslant j \leqslant k_{i}-1\right)$ be bounded measurable nonnegative functions on [0,1]. Let

$$
f=\left(f_{0} \cdot f_{\infty} \cdot \prod_{i=1}^{r} \prod_{j=1}^{k_{i}-1} f_{i j}\right)^{1 /(2 m)}
$$

For $i=1,2, \ldots, r$, denote

$$
P_{i}=g\left(x_{0}, x_{i 1}\right)\left(\prod_{j=2}^{k_{i}-1} g\left(x_{i, j-1}, x_{i, j}\right)\right) g\left(x_{i, k_{i}-1}, x_{\infty}\right) \prod_{j=1}^{k_{i}-1} f_{i, j}\left(x_{i, j}\right) .
$$

We need to prove $I \geqslant J^{m}$, where

$$
I=\int_{[0,1]^{n}} f_{0}\left(x_{0}\right) f_{\infty}\left(x_{\infty}\right) \prod_{i=1}^{r} P_{i} d \mu^{n}, \quad J=\int_{[0,1]^{2}} f(x) g(x, y) f(y) d \mu^{2} .
$$

We can assume that $f_{0}=f_{\infty}$, and $f_{i, j}=f_{i, k_{i}-j}$ for all $i$ and $j$. Indeed, we could swap $f_{0}$ with $f_{\infty}$, and $f_{i, j}$ with $f_{i, k_{i}-j}$ for all $i, j$ (this would not change the value of $I$ ), and then apply the Cauchy-Schwarz inequality to the geometric mean of both expressions for $I$.

For $i=1,2, \ldots, s$ (that is when $k_{i}$ is odd), we can add a variable $z_{i}$ and replace $g\left(x_{i,\left(k_{i}-1\right) / 2}, x_{i,\left(k_{i}+1\right) / 2}\right)$ in the expression for $P_{i}$ with the product of $h\left(x_{i,\left(k_{i}-1\right) / 2}, z_{i}\right)$ and $h\left(x_{i,\left(k_{i}+1\right) / 2}, z_{i}\right)$ :

$$
\begin{aligned}
P_{i}=g\left(x_{0}, x_{i, 1}\right)( & \left(\prod_{j=2}^{\left(k_{i}-1\right) / 2} g\left(x_{i, j-1}, x_{i, j}\right)\right) h\left(x_{i,\left(k_{i}-1\right) / 2}, z_{i}\right) h\left(x_{i,\left(k_{i}+1\right) / 2}, z_{i}\right) \\
& \times\left(\prod_{j=\left(k_{i}+3\right) / 2}^{k_{i}-1} g\left(x_{i, j-1}, x_{i, j}\right)\right) g\left(x_{i, k_{i}-1}, x_{\infty}\right) \prod_{j=1}^{k_{i}-1} f_{i, j}\left(x_{i, j}\right) .
\end{aligned}
$$

Now in the expression for $I$ we have to integrate over $n+s$ variables. Define for $i \leqslant s$,

$$
Q_{i}=g\left(x_{0}, x_{i 1}\right) \prod_{j=2}^{\left(k_{i}-1\right) / 2} g\left(x_{i, j-1}, x_{i, j}\right) h\left(x_{i,\left(k_{i}-1\right) / 2}, z_{i}\right) \prod_{j=1}^{\left(k_{i}-1\right) / 2} f_{i, j}\left(x_{i, j}\right),
$$

and for $i>s$,

$$
Q_{i}=g\left(x_{0}, x_{i, 1}\right) \prod_{j=2}^{k_{i} / 2} g\left(x_{i, j-1}, x_{i, j}\right) \prod_{j=1}^{k_{i} / 2-1} f_{i, j}\left(x_{i, j}\right)\left(f_{i, k_{i} / 2}\left(x_{i, k_{i} / 2}\right)\right)^{1 / 2} .
$$

Let $A\left(z_{1}, \ldots, z_{s}, x_{s+1, k_{s+1} / 2}, \ldots, x_{r, k_{r} / 2}\right)$ denote the integral of $f_{0}\left(x_{0}\right) \prod_{i=1}^{r} Q_{i}$ over all variables except $z_{1}, \ldots, z_{s}, x_{s+1, k_{s+1} / 2}, \ldots, x_{r, k_{r} / 2}$. Set

$$
\begin{aligned}
B\left(y_{1}, \ldots, y_{s},\right. & \left.x_{s+1, k_{s+1} / 2}, \ldots, x_{r, k_{r} / 2}\right) \\
= & \int_{[0,1]^{s}} A\left(z_{1}, \ldots, z_{s}, x_{s+1, k_{s+1} / 2}, \ldots, x_{r, k_{r} / 2}\right) \prod_{i=1}^{s} h\left(y_{i}, z_{i}\right) d \mu\left(z_{1}\right) \cdots d \mu\left(z_{s}\right) .
\end{aligned}
$$


Then

$$
I=\int_{[0,1]^{r}} A\left(z_{1}, \ldots, z_{s}, x_{s+1, k_{s+1} / 2}, \ldots, x_{r, k_{r} / 2}\right)^{2} d \mu^{r}
$$

and

$$
\begin{aligned}
I \cdot J^{s} & =I \cdot \int_{[0,1]^{s}} \prod_{i=1}^{s}\left(\int_{[0,1]} f\left(y_{i}\right) h\left(y_{i}, z_{i}\right) d \mu\left(y_{i}\right)\right)^{2} d \mu\left(z_{1}\right) \cdots d \mu\left(z_{s}\right) \\
& \geqslant\left(\int_{[0,1]^{r}} B\left(y_{1}, \ldots, y_{s}, x_{s+1, k_{s+1} / 2}, \ldots, x_{r, k_{r} / 2}\right) d \mu^{r}\right)^{2} .
\end{aligned}
$$

Notice that $\int_{[0,1]^{r}} B d \mu^{r}$ is the left hand side of (1.3) for the tree $G$ formed by paths of length $\left(k_{1}+1\right) / 2, \ldots,\left(k_{s}+1\right) / 2, k_{s+1} / 2, \ldots, k_{r} / 2$ connected at one endpoint. By Corollary 6.4 , $G$ is extra-good, so $\int_{[0,1]^{r}} B d \mu^{r} \geqslant J^{\mathrm{e}(G)}$. As e $(G)=(m+s) / 2$, we get

$$
I \geqslant J^{-s}\left(\int_{[0,1]^{r}} B d \mu^{r}\right)^{2} \geqslant J^{m}
$$

Proof of Theorem 6.6. Let $G_{0}$ be formed by attaching a $k$-edge cycle $\left(v_{0}, v_{1}, \ldots, v_{k-1}\right.$, $\left.v_{k}=v_{0}\right)$ to a vertex $v$ of graph $G$, so $v=v_{0}=v_{k}$. Let $g$ be a doubly nonnegative function. There exists $h \in \mathcal{H}$ such that $g(x, y)=\int_{[0,1]} h(x, z) h(y, z) d \mu(z)$. Assign bounded measurable nonnegative functions on $[0,1]$ to all vertices of $G$, and denote by $F(x)$ the integral on the left hand side of (1.3) taken over all variables except the one that corresponds to $v$. Assign bounded measurable nonnegative functions $f_{1}, f_{2}, \ldots, f_{k-1}$ to vertices $v_{1}, v_{2}, \ldots, v_{k-1}$. For functions $\gamma_{1}, \gamma_{2}, \ldots, \gamma_{r}$, denote

$$
I_{r}\left(x_{0}, x_{r} ; \gamma_{1}, \gamma_{2}, \ldots, \gamma_{r}\right)=\int_{[0,1]^{r-1}} \prod_{i=1}^{r}\left(g\left(x_{i-1}, x_{i}\right) \gamma_{i}\left(x_{i}\right)\right) d \mu\left(x_{1}\right) \cdots d \mu\left(x_{r-1}\right) .
$$

Let $I_{G_{0}}$ be the value of the integral on the left hand side of (1.3) for $G_{0}$. Then

$$
I_{G_{0}}=\int_{[0,1]} F(x) I_{k}\left(x, x ; f_{1}, f_{2}, \ldots, f_{k-1}, 1\right) d \mu(x) .
$$

Let $f_{0}$ be the product of all $\mathrm{v}\left(G_{0}\right)$ functions assigned to vertices of $G_{0}$. We need to prove $I_{G_{0}} \geqslant J^{\mathrm{e}\left(G_{0}\right)}$, where $J=\int_{[0,1]^{2}} f(x) g(x, y) f(y) d \mu^{2}$ and $f=\left(f_{0}\right)^{1 /\left(2 \mathrm{e}\left(G_{0}\right)\right)}$.

Set $\gamma_{i}=\sqrt{f_{i} f_{k-i}}$, so $\gamma_{k-i}=\gamma_{i}$. By the Cauchy-Schwarz inequality,

$$
\begin{aligned}
I_{k}\left(x, x ; f_{1}, f_{2}, \ldots, f_{k-1}, 1\right)^{2} & =I_{k}\left(x, x ; f_{1}, f_{2}, \ldots, f_{k-1}, 1\right) \cdot I_{k}\left(x, x ; f_{k-1}, f_{k-2}, \ldots, f_{1}, 1\right) \\
& \geqslant I_{k}\left(x, x ; \gamma_{1}, \gamma_{2}, \ldots, \gamma_{k-1}, 1\right)^{2} .
\end{aligned}
$$

If $k=2 a$, then

$$
\begin{aligned}
I_{2 a}\left(x, x ; \gamma_{1}, \ldots, \gamma_{2 a-1}, 1\right) & =\int_{[0,1]} I_{a}\left(x, y ; \gamma_{1}, \ldots, \gamma_{a-1}, \sqrt{\gamma_{a}}\right)^{2} d \mu(y) \\
& \geqslant\left(\int_{[0,1]} I_{a}\left(x, y ; \gamma_{1}, \ldots, \gamma_{a-1}, \sqrt{\gamma_{a}}\right) d \mu(y)\right)^{2} .
\end{aligned}
$$


Construct graph $G_{a}$ from graph $G$ by attaching two disjoint $a$-edge paths to vertex $v$. By Theorem 6.3, $G_{a}$ is extra-good. Assign functions $\gamma_{1}, \ldots, \gamma_{a-1}, \sqrt{\gamma_{a}}$ to vertices of each of the two paths. Let $I_{G_{a}}$ be the value of the integral on the left hand side of (1.3) for $G_{a}$. Then

$$
I_{G_{a}}=\int_{[0,1]} F(x)\left(\int_{[0,1]} I_{a}\left(x, y ; \gamma_{1}, \ldots, \gamma_{a-1}, \sqrt{\gamma_{a}}\right) d \mu(y)\right)^{2} d \mu(x) \leqslant I_{G_{0}} .
$$

The right hand side of (1.3) is the same for both $G_{0}$ and $G_{a}$, so we are done with the case $k=2 a$. If $k=2 a+1$, we have

$$
\begin{aligned}
& I_{2 a+1}\left(x, x ; \gamma_{1}, \ldots, \gamma_{2 a}, 1\right) \\
& =\int_{[0,1]^{2}} I_{a}\left(x, y^{\prime} ; \gamma_{1}, \ldots, \gamma_{a}\right) g\left(y^{\prime}, y^{\prime \prime}\right) I_{a}\left(x, y^{\prime \prime} ; \gamma_{2 a}, \ldots, \gamma_{a+1}\right) d \mu\left(y^{\prime}\right) d \mu\left(y^{\prime \prime}\right) \\
& \quad=\int_{[0,1]}\left(I_{a}\left(x, y ; \gamma_{1}, \ldots, \gamma_{a}\right) h(y, z) d \mu(y)\right)^{2} d \mu(z),
\end{aligned}
$$

and

$$
J=\int_{[0,1]^{2}} f\left(s^{\prime}\right) g\left(s^{\prime}, s^{\prime \prime}\right) f\left(s^{\prime \prime}\right) d \mu^{2}=\int_{[0,1]}\left(\int_{[0,1]} h(s, z) f(s) d \mu(s)\right)^{2} d \mu(z) .
$$

Hence, by the Cauchy-Schwarz inequality,

$$
\begin{aligned}
& I_{2 a+1}\left(x, x ; \gamma_{1}, \ldots, \gamma_{2 a}, 1\right) \cdot J \geqslant \\
& \geqslant\left(\int_{[0,1]^{3}} I_{a}\left(x, y ; \gamma_{1}, \ldots, \gamma_{a}\right) h(y, z) h(s, z) f(s) d \mu(y) d \mu(z) d \mu(s)\right)^{2} \\
& =\left(\int_{[0,1]} I_{a+1}\left(x, s ; \gamma_{1}, \ldots, \gamma_{a}, f\right) d \mu(s)\right)^{2} .
\end{aligned}
$$

Construct $G_{a+1}$ from $G$ by attaching two disjoint $(a+1)$-edge paths to vertex $v$. By Theorem 6.3, $G_{a+1}$ is extra-good. Assign functions $\gamma_{1}, \ldots, \gamma_{a}, f$ to vertices of each of the two paths. Let $I_{G_{a+1}}$ be the value of the integral on the left hand side of (1.3) for $G_{a+1}$. Then

$$
I_{G_{a+1}}=\int_{[0,1]} F(x)\left(\int_{[0,1]} I_{a+1}\left(x, s ; \gamma_{1}, \ldots, \gamma_{a}, f\right) d \mu(s)\right)^{2} d \mu(x) \leqslant I_{G_{0}} \cdot J .
$$

The right hand side of $(1.3)$ is equal to $J^{\mathrm{e}\left(G_{0}\right)}$ for $G_{0}$, and $J^{\mathrm{e}\left(G_{a+1}\right)}=J \cdot J^{\mathrm{e}\left(G_{0}\right)}$ for $G_{a+1}$. As (1.3) holds for $G_{a+1}$, it holds for $G_{0}$, too. 